\title{
Perceptual grouping in Gabor lattices: Proximity and alignment
}

\author{
PETER M. CLAESSENS and JOHAN WAGEMANS \\ University of Leuven, Leuven, Belgium
}

\begin{abstract}
We propose the Gabor lattice as a new stimulus designed to deal with multiple organizations in perceptual grouping, allowing both comparison between psychophysical data and neural findings and a systematic investigation of grouping based on several low-level characteristics and their interactions. A Gabor lattice is a geometric lattice with Gabor patches, evoking a multistable global orientation percept. Visual grouping in Gabor lattices with elements aligned in a global orientation was compared with grouping of nonaligned Gabor patches and of Gaussian blobs. The effect sizes of proximity and alignment were estimated in logistic regression analyses. The results confirmed the importance of proximity and local element alignment as factors in dynamic grouping. We also found a small but consistent enhancement of grouping along the global vector orthogonal to the local patch orientations. In light of these results, we further motivate the relevance of these stimuli and the associated experimental paradigm.
\end{abstract}

From retinal input, the visual system has to infer the identities and locations of objects with which we might interact in a motoric or cognitive way. To successfully accomplish this task, the amorphous information mosaic needs to be ordered so that coherent regions and lines that will provide candidates for object surfaces and boundaries can be retrieved. Phenomenological demonstrations within the framework of Gestalt psychology have been an important source of inspiration for later psychophysical and neurophysiological experiments investigating the rules of this process of early, precognitive parsing. Unlike in the heyday of Gestalt psychology, the means to unravel the mechanisms underlying grouping and segmentation are now available. A full explanation of the multitude of phenomena and experimental results in this domain requires a converging movement of the several disciplines involved. The present article discusses a stimulus composed of elements specifically aimed at matching wellknown properties of the neural basis of the visual system within a psychophysical experimental methodology.

Grouping problems occur at many different spatial scales, from bridging interruptions in the retinal image caused by blood vessels to wide occlusions of distal stimuli by nearby objects. Therefore, it is not surprising that the visual system does not rely on one single mechanism to resolve occlusions and other forms of grouping ambi-

This research was supported by the Fund for Scientific Research, Flanders (Grant G.0095.03). We thank Karl Delbarge for his assistance in data collection, as well as Peter Zaenen, Filip Germeys, and Jan Vanrie for reading and commenting on the manuscript. We are also grateful for the comments of two anonymous reviewers and of Todd Maddox. Correspondence relating to this article may be sent to J. Wagemans, University of Leuven, Laboratory of Experimental Psychology, Tiensestraat 102, B3000 Leuven, Belgium (e-mail: johan.wagemans@psy.kuleuven.ac.be). guities in its input. Watt and Phillips (2000) distinguished between prespecified and dynamic groupings. In the prespecified type, grouping is a consequence of converging input in the system hierarchy. It is prespecified in that grouping procedures in this category will always converge toward the same solution for a given input. An example of this principle - simplified here for argument's sake — can be found in the earliest stages of image processing. Retinal ganglion cells project to the lateral geniculate nucleus, from which local orientation information is extracted by the appropriate pattern of projections to V1. This hardwired structure, which results in local orientation selectivity known as the receptive field of orientation-selective neurons, can be considered an early implementation of grouping of local retinal signals.

\section{Current Approaches to Grouping}

Since it became obvious that the visual system does not merely propagate local measurements in a strictly feedforward fashion, the notion of a "receptive field" has become more fuzzy (see Fitzpatrick, 2000, for a review). A class of experiments with line segments (see, e.g., Dresp, 1999; Tzevanov \& Dresp, 2002) and Gabor patches (reviewed by Polat, 1999) definitively refuted the idea of the primary projection region as an array of passive localized filters. Polat and Sagi $(1993,1994)$ have shown that detection of a low-contrast Gabor patch in a two-alternative forced choice (2AFC) paradigm is facilitated or inhibited by flanking Gabor patches well outside the classical receptive field of an orientation-selective cell in V1. The sign and magnitude of the modulation is dependent on both the distance of the target to the flankers and the global configuration of the stimulus.

Psychophysical findings in this line of research are supported by neurophysiological methods in experiments 
involving visually evoked potential recordings in humans (Polat \& Norcia, 1996), single-cell recordings in cat striate cortex (Polat, Mizobe, Pettet, Kasamatsu, \& Norcia, 1998), and single-cell recordings of V1 complex cells in macaque monkeys (Kapadia, Ito, Gilbert, \& Westheimer, 1995; Kapadia, Westheimer, \& Gilbert, 2000). In general, the distance, lateral offset, and angular offset of flanker and target weaken threshold facilitation. These and similar findings have played an important role in establishing the modalities of interactions beyond the extent of the receptive field in both the psychophysical and the neurophysiological domains.

Although these experiments have explored how contextual information modulates local features such as perceived contrast of an element, there has also been great interest in the role of local features on contour integration. In a classical paradigm, a dotted line is embedded in a background of randomly placed dots in order to investigate the detection of linear structure for different levels of proximity and collinearity (Beck, Rosenfeld, \& Ivry, 1989; Smits, Vos, \& Van Oeffelen, 1985; Uttal, 1975). Field, Hayes, and Hess (1993) applied the same principle in the construction of their stimuli. A set of Gabor patches aligned along a smoothly undulating curve was embedded in a noise background of randomly oriented Gabor elements. Subject performance was measured as a function of interelement angle in a $2 \mathrm{AFC}$ paradigm. This experimental approach has proven very popular in subsequent studies (reviewed by Hess \& Field, 1999, and Hess, Hayes, \& Field, 2003) in which a large number of local or global stimulus properties have been assessed, such as contour closure (Kovacs \& Julesz, 1993), polarity and luminance contrast along the path (Field, Hayes, \& Hess, 2000), chromaticity and color contrast (Beaudot \& Mullen, 2003; McIlhagga \& Mullen, 1996), spatial frequency tuning (Dakin \& Hess, 1998), eccentricity (Hess \& Dakin, 1999; Nugent, Keswani, Woods, \& Peli, 2003), temporal properties of the presentation (Beaudot, 2002; Hess, Beaudot, \& Mullen, 2001), and stationary motion (Bex, Simmers, \& Dakin, 2001). Most relevant in the context of this article is the consistent finding that contour saliency increases with element alignment and proximity. Field et al. (1993) advocated the existence of an association field as a constraint to contour integration processes. According to this concept, the facilitation of nearby local orientation filters propagates along a path favoring smooth contours. The connection weight between local orientation channels thus declines with length and curvature of the (virtual) contour fragment connecting them. The emergence of the association field is thought to reflect statistical regularities in the visual environment, which in turn seem to arise from the good-continuation constraint (Elder \& Goldberg, 2002; Sigman, Cecchi, Gilbert, \& Magnasco, 2001).

In general, the psychophysical results and the neurophysiological measurements in both contrast enhancement and "snake detection" experiments are qualitatively well explained in modeling efforts (among others, by Usher, Bonneh, Sagi, \& Herrmann, 1999, and Pettet, McKee,
\& Grzywacz, 1998, respectively) motivated by recent discoveries in cortical architecture. V1 pyramidal cells in macaque cortex have axon collaterals that reach up to eight hypercolumns, integrating information over distances that are multiples of classical receptive-field size (Gilbert, 1992; Stettler, Das, Benett, \& Gilbert, 2002). In the superficial layers of primary visual cortex, these horizontal connections are mostly orientation specific. This long-range connectivity has a prominent place in the discussion about the machinery of contour detectionmodulating processes, as found in psychophysical and neurophysiological experiments (Gilbert, Das, Ito, Kapadia, \& Westheimer, 1996). Extrapolating from findings in the visual cortex of the tree shrew, some researchers have suggested that these horizontal projections in V1 might extend preferentially to cortical sites along the elongation axis of the receptive fields they radiate from (Bosking, Zhang, Schofield, \& Fitzpatrick, 1997; Chisum, Mooser, \& Fitzpatrick, 2003).

\section{The Multistable Lattice Paradigm as a Complementary Approach}

This set of psychophysical, neurophysiological, and neuroanatomical results converges into a fairly unified, consistent view on contextual processing and the substrates for grouping. Yet, we believe that an approach based on Gabor lattices will offer some new methodological possibilities and elicit novel questions. (1) Despite quite a bit of research designed to give experimental support to Gestalt laws, a lot remains to be discovered about how they combine. We feel that an approach based on stimuli in which different organization principles can lead to multiple solutions rather than one has a great potential to solve questions about cooperative and competitive interactions between organization principles. In the

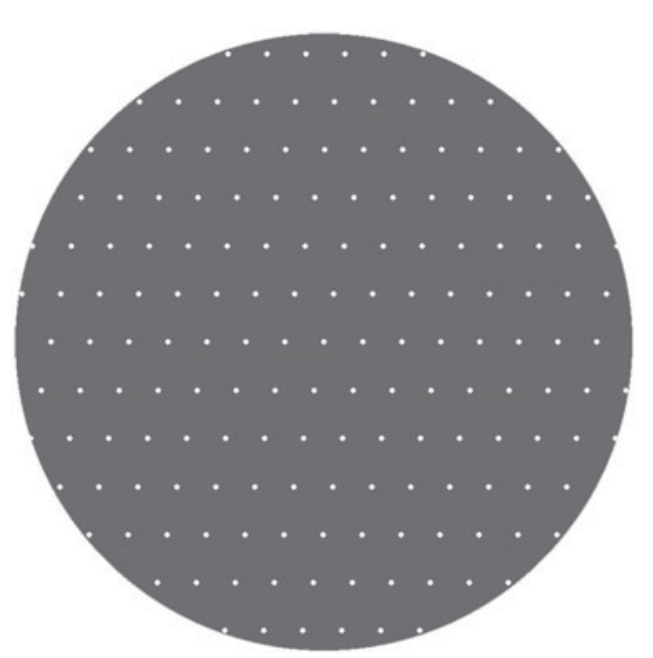

Figure 1. Example of an oblique dot lattice (after Kubovy \& Wagemans, 1995). Spontaneous visual grouping clusters the dots in lines, with proximity as the crucial factor determining the odds of organizing the lattice in one or the other orientation. 


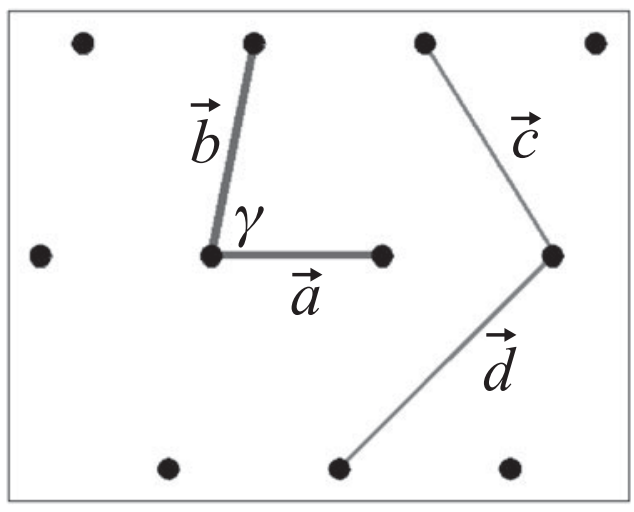

Figure 2. Parameters of the basic parallelogram spanning a point lattice.

Gabor snake detection paradigm, facilitation or inhibition of organization can only be evaluated relative to good continuation, simply because that is what defines the "signal." (2) The basic detection paradigm in which a Gabor snake is embedded in noise elements is well suited to reveal how a scene consisting of a single contour is analyzed by the visual system. In a natural scene, however, multiple edges and lines - usually suprathreshold - cross and intersect. This poses another class of problems to the visual system: not whether a local orientation channel belongs to a contour or not, but which contour it belongs to. There is need for a paradigm that relates organization of Gabor stimuli explicitly to the latter issue. But how could a lattice paradigm address these two concerns?

A two-dimensional lattice is a spatial pattern consisting of elements that are laid out in a very regular fashion. The lattice elements are spaced evenly to be collinear along different orientations. In response to the first issue raised above, note that the lattice structure has the inherent advantage that the relative weights of up to three or four different factors can be pitted against each other in one presentation frame (e.g., proximity versus similarity in the studies of Ben-Av \& Sagi, 1995; Quinlan \& Wilton, 1998; and Zucker, Stevens, \& Sander, 1983). In the case of two factors, the stimulus can induce cooperativity between Gestalt principles when both favor the same lattice orientation. The visual system can likewise settle in one of two competitive lattice vectors if the underlying grouping forces are in opposition. As to the second issue, the percept of a sufficiently entropic lattice is not prespecified; this is known as the multistability property (Kubovy \& Wagemans, 1995). Although several contour candidates are simultaneously present as the different lattice vectors, the lattice structure elicits a single emergent global orientation. This global percept is the macroscopic, conscious consequence of the identification of the relations between elements - their "belonging together"-rather than of the mere presence of a contour. The issue can be compared with the difference between the representation of a curve in itself and the representation of which of the curve parts belong to one unit-in other words, between contour rep- resentation and "curve tracing" (Roelfsema, Lamme, \& Spekreijse, 1998).

In addition to the benefits discussed above, we think that the use of lattices has additional value. (1) The findings with Gabor patterns we reviewed above do not readily explain an important phenomenological aspect of lattice stimuli: The suggested process of parallel computation across the visual field does not result in independent local organizations. In other words, it is not obvious how locally operating association fields of the type proposed by Field et al. (1993) could lead to a single dominant orientation that is consistent across the entire stimulus. More generally stated, little is known or modeled about the properties of perceptual organization with Gabor stimuli beyond the scope of nearest-neighbor patches, and we believe that Gabor lattices can contribute to filling that gap. (2) Any explanation that relies on contrast enhancement to explain the emergence of a salient orientation (such as in the model by Pettet et al., 1998) fails to explain the saliency experienced in Gabor lattices.

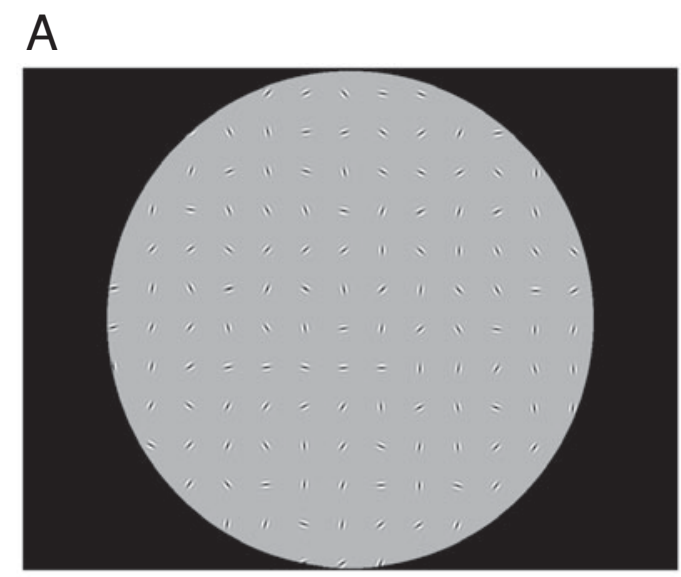

B

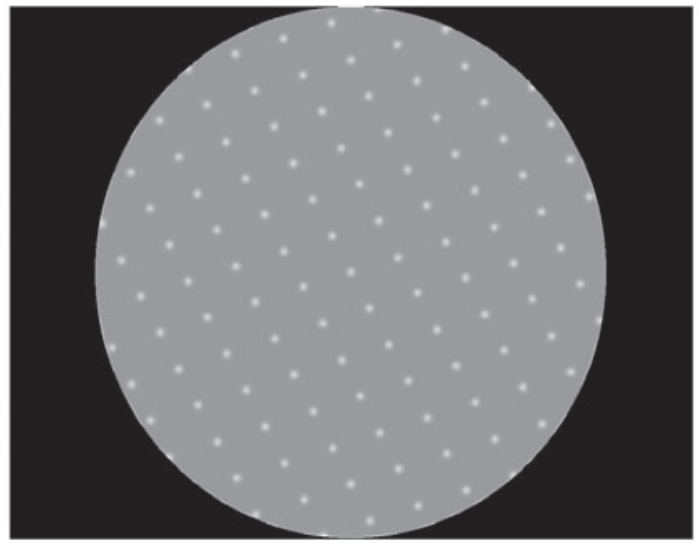

Figure 3. Examples of control conditions. (A) Example of a Gabor lattice without alignment, with a rectangular lattice structure. The orientation of each Gabor element is random; apparent local collinearities or parallelisms are simply by-products of the statistical properties of randomly generated orientations. (B) Example of a Gauss blob lattice, with an oblique lattice structure. 


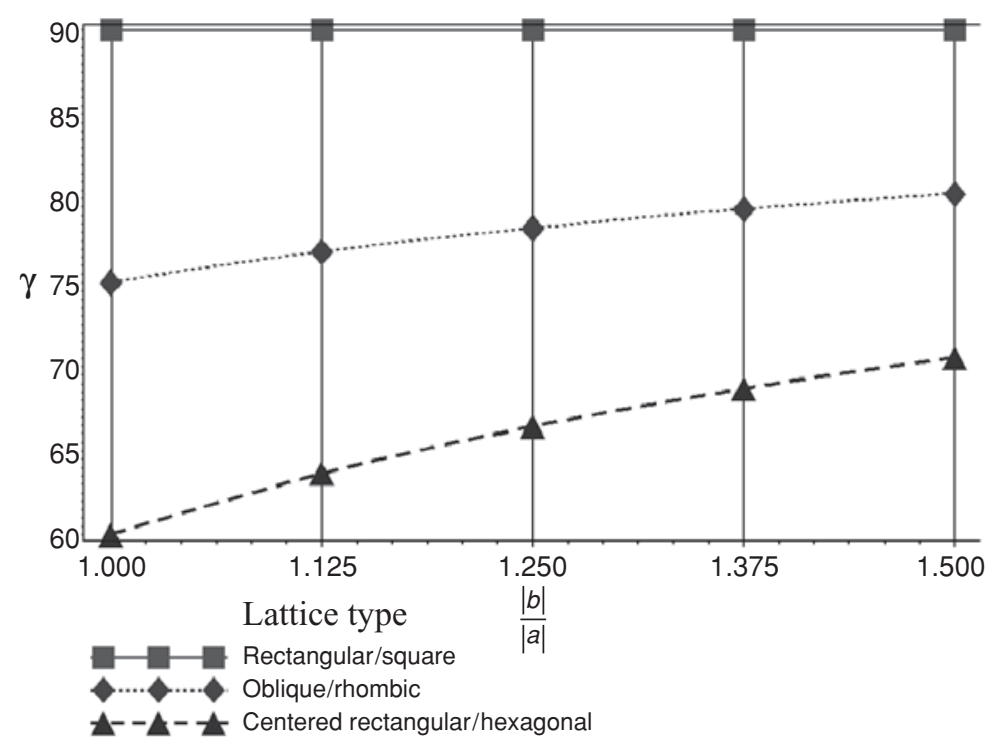

Figure 4. Sampling of the lattice space. Values of $\gamma$ were set at $90^{\circ}$ (rectangular lattices, or square if $|a|=|b|$, the minimal value given a particular ratio of $|b| /|a|$ (centered rectangular, or hexagonal if $|a|=|b|$ ), and the value midway between these (oblique structure, or rhombic if $|a|=|b|$ ). The vector length ratios varied from 1.000 to 1.500 at intervals of 0.125 .

Since the local structure in a lattice is by definition invariant over the visual field, a contrast enhancement or other effect would apply to every single element and increase the perceived contrast of the stimulus as a whole. The independence of contour saliency and perceived contrast found by Hess, Dakin, and Field (1998) and Meese, Hess, and Williams (2001) supports this observation. Empirical studies employing Gabor lattice structures will help to develop extensions or alternatives to current theories of contour grouping. (3) Finally, the phenomenological multistability property creates a different perspective. It allows for making a link to the earlier work on lattices and its methods of measurement and quantification that goes beyond mere comparison of proportions correct in different conditions. It is also ideally suited to addressing questions relating to dynamical properties of the underlying processes, such as hysteresis and adaptation as applied to perceptual grouping.

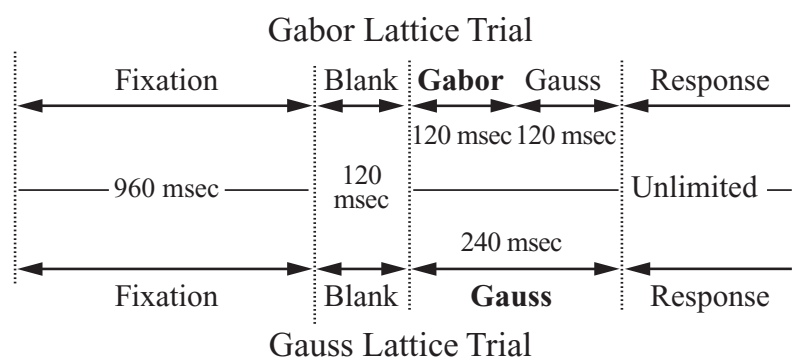

Figure 5. Schematic overview of the course of an experimental trial. Upper row: Time course for a Gabor lattice presentation. Lower row: Time course for a trial with a Gauss blob lattice.

\section{Gabor Lattices}

The psychophysical procedure used here mainly draws on work with dot lattices, a class of stimuli consisting of co-oriented rows of dots with regular spacing (see Figure 1). The space of dot lattices, the taxonomy of which dates back to the 19th century and the crystallographic work of Bravais (1866), was parameterized by Kubovy (1994). In this class of stimuli, dots are aligned in parallel lines along several orientations. Within each orientation the interelement distance is constant, but the exact value of this distance varies across orientations, depending on the lattice geometry. According to the Gestalt law of proximity, the dots tend to be perceptually grouped along the orientation in which the dots are closest (Schumann, 1900; Wertheimer, 1923/1955). A modern version of this grouping principle, adapted to account for stochastic responses, matched experimental results very well in a formal model with only one free parameter (Kubovy, Holcombe, \& Wagemans, 1998; Kubovy \& Wagemans, 1995). The quintessence of this quantitative Gestalt law can be summarized as follows: Each lattice orientation has an associated grouping strength that exponentially decays with interdot distance (the attraction function); the probability of organizing the dot lattice in a specific orientation is given by this grouping strength relative to the sum of attraction forces present in the lattice. With all relative distances taken into account, no evidence for an additional role of configuration was found, and consequently the distance-grouping relation that has been inferred from experiments involving dot lattices is referred to as the pure distance law.

In the present study, we made use of lattice stimuli composed of Gabor elements rather than dots for several 


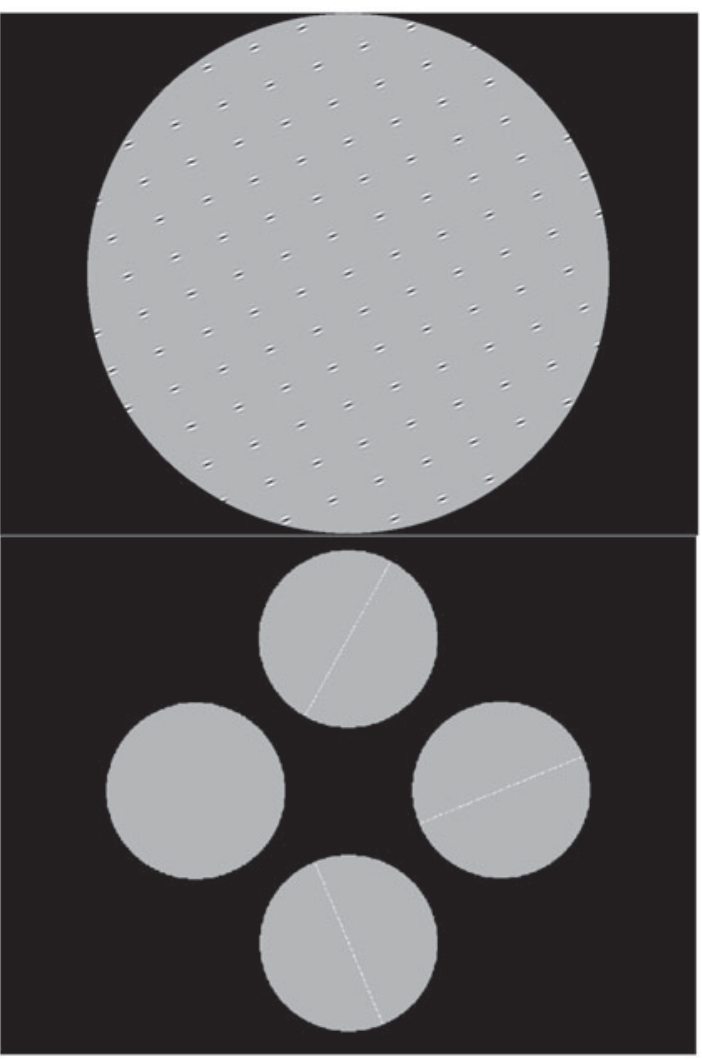

Figure 6. Upper panel: Example of a stimulus display (rectangular Gabor lattice, $b$ aligned). Lower panel: Corresponding response display.

reasons: (1) The lawfulness of proximity grouping in dot lattice structures has been demonstrated and replicated. The use of Gabor elements allowed for measurement and quantification of additional grouping principles, such as good continuation through the alignment of local orientations. (2) Gabor patches carry local information for several well-studied dimensions - spatial position, orientation, elongation, frequency, and phase - that can be parameterized and manipulated systematically. (3) To aid understanding of the implementation of grouping laws in the human visual system, it is desirable that stimulus elements reflect receptive field properties of early processing stages in visual cortex. Since the receptive field properties of orientation-selective simple cells in V1 can be described by a Gabor function (see, e.g., Marcelja, 1980), and given that the Gabor function is also justified by formal arguments (e.g., Daugman, 1985), it seems appropriate to use matching luminance profiles in order to have at least a generalized idea of how the stimulus is initially encoded in the striate areas of the visual cortex.

\section{METHOD}

\section{Subjects}

Both authors and a research assistant were submitted to 10 sessions each (i.e., a total of 11,250 trials per subject; see the Proce- dure section below), and 5 naive observers, all of them volunteering students, participated in 2 sessions each (i.e., a total of 2,250 trials apiece). All subjects had normal or corrected-to-normal vision.

\section{Stimuli}

The geometric structure underlying the patterns used in the experiment is the point lattice. A classical point lattice is a two-dimensional periodic structure consisting of points spread over the plane, invariant under specific translations. The pattern can be characterized by the parameters of the basic parallelogram that spans the lattice (see Figure 2). The vectors constituting the sides of the parallelogram are the shortest distances between elements in the lattice and are denoted $\mathbf{a}$ and $\mathbf{b}$, with an intervector angle $\gamma$. The vector difference and sum of $\mathbf{a}$ and $\mathbf{b}$, which are the diagonals of the basic parallelogram, are denoted $\mathbf{c}$ and $\mathbf{d}$, respectively. Combination of the $|b| /|a|$ and $\gamma$ parameters defines a restricted space of lattices in which six lattice types are embedded (Kubovy, 1994). The several lattice types differ in the number and nature of the visual symmetries present in the stimulus geometry. The taxonomy derives from the most regular polygon leading to the lattice layout when tiled: square, rhombic, or hexagonal when $|a|=|b|$, and rectangular, oblique, or centered rectangular when $|a|<|b|$.

As in the parametric dot lattice research initiated by Kubovy and Wagemans (1995), lattices in the present study were presented in random orientations, with $\gamma$ randomly set either clockwise or counterclockwise with respect to a. These procedures constitute a clear improvement over the methods in the classic Gestalt demonstrations and the older psychophysical literature, in which only square and rectangular lattices were shown in a vertical orientation. An impression of looking through a circular aperture was evoked so as to reduce the interaction with external orientation cues (see Figure 1).

In order to include orientation alignment as a grouping cue, and for the reasons mentioned in the introduction, Gabor patches were used rather than dots. A two-dimensional Gabor function is characterized by the parameters of a Gauss function (amplitude, location, and spread) and by those of the sine function it is multiplied with (period and phase). The local orientation of the Gabor elements could be aligned with the $\mathbf{a}, \mathbf{b}$, or $\mathbf{c}$ vector of the lattices. Gabor patches could also be independently rotated to a random orientation (i.e., a nonaligned condition; see Figure 3A). We did not align the patches along the orientation of the $\mathbf{d}$ vector, since analysis of previous results showed that corresponding responses often result from mistaking the d-response alternative for the $\mathbf{a}$ or $\mathbf{b}$ groupings, particularly in centered rectangular lattices (Kubovy et al., 1998).

It can be expected that element alignment along a particular orientation will increase the odds of a global grouping along this orientation. To measure the magnitude of this effect, the Gabor lattices were compared with stimuli in which the elements had no intrinsic orientation. The lattice elements in this control condition only reflected the Gauss function, without the sine component (see Figure 3B). We used matched values for the spread parameter with Gauss blobs and Gabor patches.

In this study, the parameters of the localized elements were fixed. We chose to use a radially symmetric Gaussian profile with a spread $\sigma$ of four pixels and a sine period of eight pixels for the Gabor patches. At a viewing distance of approximately $90 \mathrm{~cm}$, the latter was equivalent to $9^{\prime}$ of visual angle. Applying a sine phase of $-90^{\circ}$, we obtained Gabor patches centered around a luminance minimum (even symmetric, off center). With identical values for corresponding parameters, the center of the Gaussian dots was a luminance maximum. ${ }^{1}$ With these parameter values, we obtained Gabor patches with a luminance range between approximately 0.5 and $80 \mathrm{~cd} / \mathrm{m}^{2}$ and low-contrast Gauss blobs with a maximum of approximately $40 \mathrm{~cd} / \mathrm{m}^{2}$, both presented against a background of $32 \mathrm{~cd} / \mathrm{m}^{2}$.

Figure 4 illustrates how the lattice space was systematically sampled for five levels of $|b| /|a|$, from 1 to 1.5 , with three levels of the intervector angle $\gamma$ per distance ratio. Scaling remained fixed at $|a|$ 


\section{Rectangular}
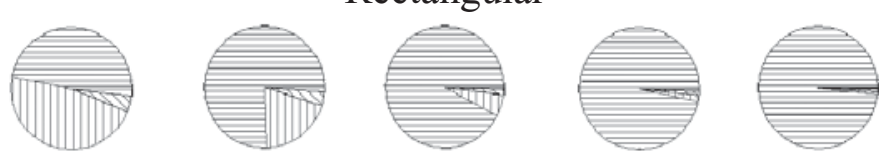

Oblique
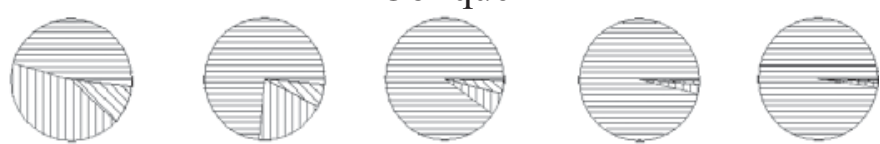

Centered Rectangular
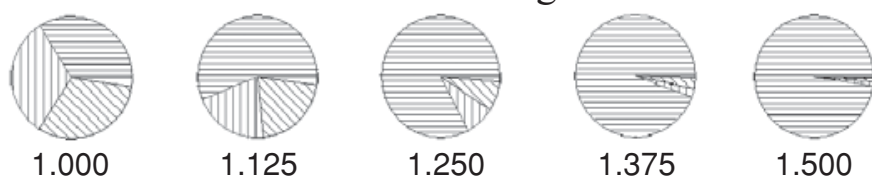

1.000

1.125

1.250

1.375

1.500
a responses
b responses
c responses
Blank responses

Figure 7. Response frequencies aggregated over subjects for the Gauss blob lattices. The pie charts are arranged in the same order as the positions that they correspond to in the lattice space (see Figure 4) - that is, with increasing levels of $|b| /|a|$ from left to right and with levels of $\gamma$ increasing to $90^{\circ}$ from the bottom to the top. For increasing values of $|b|$, and thus of $|b| /|a|$, the probability of organization along the a vector increases. Similarly, the likelihood of c-oriented organization decreases for higher values of $\gamma$, which for geometric reasons yield higher values of $|c|$.

equal to 60 pixels, or $1^{\circ}$ of visual angle. The viewing "aperture" had a radius of 380 pixels, or about $14^{\circ}$ diameter.

In sum, we used 15 different lattice configurations, crossed with 5 stimulus types - Gabor lattices either aligned along $\mathbf{a}, \mathbf{b}$, or $\mathbf{c}$ or nonaligned, and Gauss lattices - resulting in 75 stimulus conditions.

\section{Apparatus}

Stimuli were presented on a 17 -in. noninterlaced CRT driven by an S3 Vision 968 PCI graphics adapter and a 120-MHz Pentium PC. The experiment was run, at a screen resolution of 1,024 horizontal $\times 768$ vertical pixels and a vertical refresh rate of $70 \mathrm{~Hz}$, by an MSDOS real-mode application.

\section{Procedure}

The dot lattice paradigm typically involves brief lattice exposures, after which the subjects indicate the grouping orientation that emerged from the lattice presentation. Pilot experiments (Wagemans, Claessens, Delbarge, Eycken, \& Kubovy, 1999; Wagemans, Eycken, Claessens, \& Kubovy, 1999) showed that with a classical presentation duration of $300 \mathrm{msec}$, some subjects tended to focus on the orientation of a central patch instead of establishing a global organization. Apparently evaluation of a physical local orientation is easier, and thus requires less effort and attention, than deriving a virtual global orientation. To decrease the saliency of local orientation information so as to avoid task confusion among the naive subjects, we decided to make the local orientation information available only very briefly. After $120 \mathrm{msec}$, the Gabor patches were replaced by Gauss blobs that remained on screen for $120 \mathrm{msec}$, thus preserving proximity information. In the conditions with Gauss lattices, the stimulus remained unchanged for a total duration of $240 \mathrm{msec}$. The actual stimulus presentation was preceded by a $960-\mathrm{msec}$ presentation of the circular window with a central fixation dot, followed by a blank disk for $120 \mathrm{msec}$ (see Figure 5). Immediately after the presentation of the lattice, the response display was shown (see Figure 6). It contained four circles: three disks with lines representing the most likely grouping alternatives (corresponding to the $\mathbf{a}, \mathbf{b}$, and $\mathbf{c}$ vectors) and a blank circle for cases in which a subject had clustered the elements along the $\mathbf{d}$ orientation or in any other way. The subjects responded by clicking with a mouse pointer on the disk in the response menu with the appropriately oriented bisecting line. After a subject had indicated his or her choice with the mouse pointer, a new trial cycle began.

Given the possible interference of local orientation with response behavior, the subjects were explicitly asked to keep their attention spread while fixating on the middle of the screen. During an experimental session, each of the 75 conditions ( 15 lattices $\times 5$ alignment manipulations) was presented 15 times. Trials were presented in a random order, with an opportunity for a break every 225 trials. Each 1,125-trial session lasted approximately $1 \mathrm{~h}$.

\section{RESULTS}

We will first present the main findings graphically and support them with logistic regression analyses. Then, an analysis of the experimental manipulations and their interactions will be provided. ${ }^{2}$

\section{Role of Proximity and Alignment}

Given the large number of conditions and response alternatives, it is not feasible to show the data for each of the conditions for each of the subjects. Figure 7 shows response frequencies per stimulus condition within the set of Gauss 


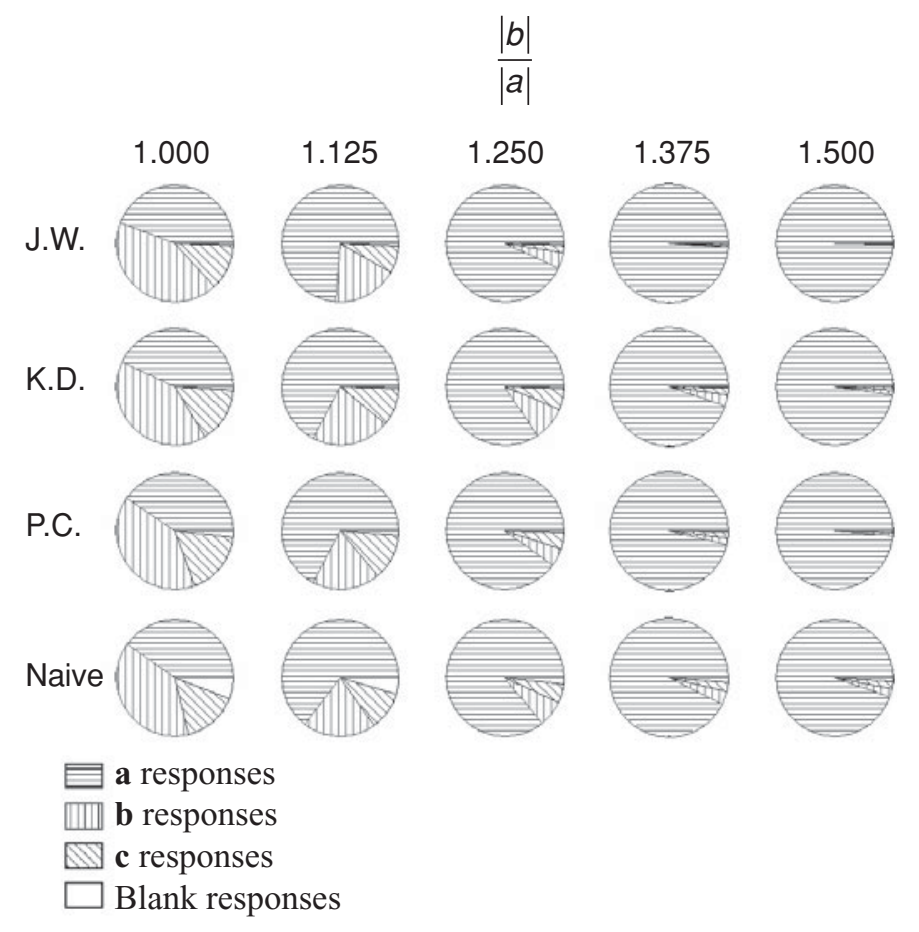

Figure 8. Effect of proximity for different observers, aggregated and averaged over $\gamma$. The response frequencies presented in the naive block are aggregated across the $\mathbf{4}$ naive subjects in order to obtain a total number of trials, and hence statistical power, of the same order of magnitude as for the expert subjects.

blob lattices relative to the position in the lattice space (i.e., a particular $|b| /|\mathrm{a}|$ and $\gamma$ ). In this summarizing graph, response frequencies are aggregated across subjects.

It is clear that lattice geometry influences the response pattern. Over lattice types, larger $|b| /|\mathrm{a}|$ values (columns in Figure 7) yield fewer b responses. This reflects the effect of the distance manipulation. Similarly, for larger values of $\gamma$ (rows in Figure 7), corresponding to larger $|c| /|a|$ ratios, the number of $\mathbf{c}$ responses decreases. These observations are congruent with previous results (Kubovy \& Wagemans, 1995) and with the principle of grouping by proximity (Kubovy et al., 1998). The results obtained with nonaligned Gabor lattices (not shown) are similar. Figure 8 (results aggregated across all alignment conditions and all $\gamma$ values) shows that the weight of proximity as a grouping factor varies somewhat across observers, although the pattern of results is identical.

In comparison with the Gauss lattices and the nonaligned Gabor lattices, the aligned Gabor lattices elicit more $\mathbf{a}$ responses in $\mathbf{a}$-aligned lattices, more $\mathbf{b}$ responses in $\mathbf{b}$-aligned lattices, and more $\mathbf{c}$ responses in $\mathbf{c}$-aligned lattices (Figure 9). This result shows that local orientation alignment acts as a grouping cue. In lattices with small differences between the $\mathbf{a}$ - and $\mathbf{b}$-vector lengths, orientation alignment along $\mathbf{b}$ could override proximity as a grouping cue in a limited range. Below, we will estimate the transition point at which orientation alignment starts dominating the grouping response.
The statistical analyses are focused on the $\mathbf{a}$ and $\mathbf{b}$ responses. Since the corresponding vectors are shortest, they generate more responses than did the $\mathbf{c}$ (and $\mathbf{d}$ ) vectors; consequently, these data are less prone to statistical floor effects. Moreover, judgment errors in selection for the response display throughout the experiment-most frequently confusion of the $\mathbf{b}, \mathbf{c}$, and $\mathbf{d}$ orientations for lattices with a high $|b| /|a|$ ratio (Kubovy et al., 1998) — have less influence on response categories with higher frequencies.

The pure distance law proposed by Kubovy and Wagemans (1995) can be simplified to a classical logistic model in which the generalized logit $\ln [p(b) / p(a)]$ is linearly dependent on $|b| /|a|-1$ (Kubovy et al., 1998). Figure 10 shows the observed log-odds as a function of the corresponding distance ratio; in Figure 11, the same data are plotted for each subject individually, with aggregated control conditions. Since b responses constitute the numerator, a higher position of a point in the graph corresponds to a higher likelihood of choosing b. As described previously, b alignment yields more $\mathbf{b}$ responses, which is why there is a positive intercept for the $\mathbf{b}$-aligned conditions with respect to the log-odds for the other lattices. Conversely, a alignment leads to more a responses, and therefore to a negative intercept for the a-aligned conditions. The lattices aligned along the $\mathbf{c}$ vector have a spline with a course coinciding with those for lattices with Gauss blobs and nonaligned Gabor patches. This indicates that, although c alignment evidently yields more $\mathbf{c}$ responses, the relative 


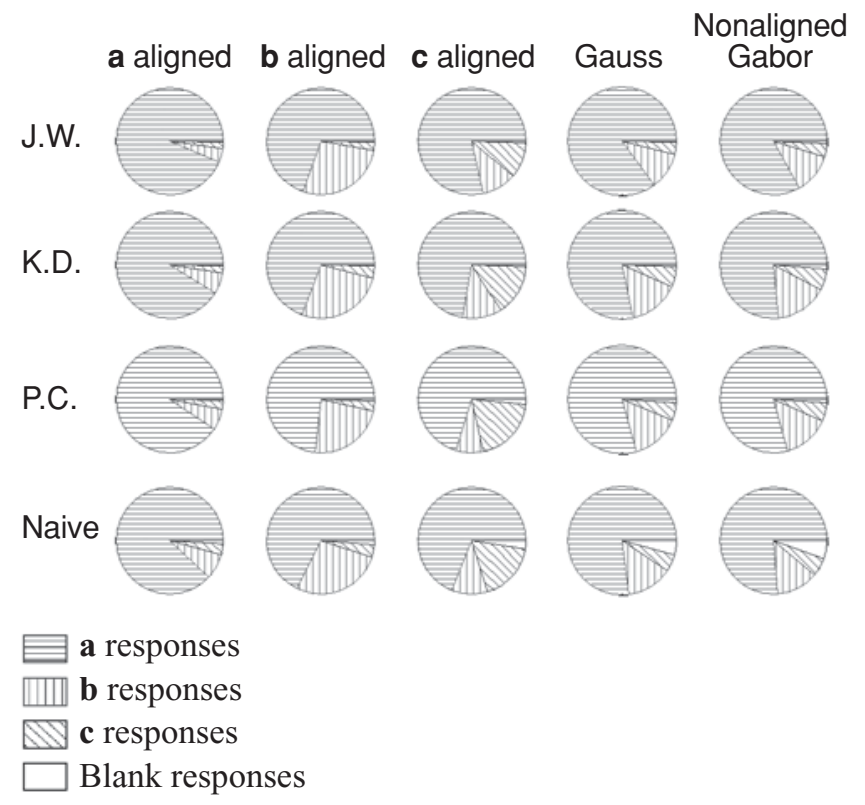

Figure 9. Influence of the alignment manipulation for the different subjects, aggregated over distance conditions and lattice types. The first three columns show the response frequencies for Gabor lattices that were aligned with the $a, b$, and c vectors, respectively. The fourth and fifth columns contain the pie charts for the control conditions: the lattices consisting of Gauss blobs ("Gauss") and the Gabor lattices in which each patch was assigned a random orientation ("Nonaligned Gabor").

proportions of $\mathbf{a}$ and $\mathbf{b}$ responses are still largely equal. This fact is reminiscent of an axiomatic idea of choice theory generally referred to as independence of irrelevant alternatives. It is stated as a probabilistic lemma in Luce (1959): The probability ratio of two choices should not depend on the set of alternatives that is under consideration. Our data seem to satisfy this condition.

There is an obvious parallelism between the splines for the conditions in which Gabor patches were used, except for the c-aligned lattices, for which - because of the lower number of $\mathbf{a}$ and $\mathbf{b}$ responses - variability is the largest (compare full lines in Figure 10). For all other conditions involving Gabor patches, whether a-aligned, b-aligned, or nonaligned, the proximity effect levels off. The graph suggests a more linear trend for the stimuli with Gauss blobs in comparison with those from the Gabor lattice conditions, in line with the analysis for dot lattices by Kubovy et al. (1998). At the individual level (Figure 11), this curvilinear trend shows up in 1 nonnaive and 3 naive subjects. The level at which the logit asymptotes, in particular for the b-aligned Gabor lattices, is quite far from the extremes in several subjects. This makes an explanation in terms of a statistical floor effect or random erratic responses unlikely. It seems to indicate instead that mechanisms for grouping diverge for Gabor elements and other stimuli when extreme distance ratios come into play. Note, however, that the curvilinear trends in different alignment conditions do not necessarily correspond to one and the same cause.

\section{Logistic Weights of Proximity and Alignment}

The $\mathbf{a}$ and $\mathbf{b}$ response frequencies were processed in logistic regression analyses in order to assess statistical significance of the visual observations above. The simplest statistical model for the logistic link function $\ln [p(b) / p(a)]$ that captures the basic characteristics of the set of splines for all of the alignment conditions contained $|b| /|a|-1$ as a numerical variable, its square $(|b| /|a|-1)^{2}$ to capture curvilinearity, and alignment as a class-level variable. Both this model and those in subsequent analyses were formulated as binary logistic models and fitted by maximumlikelihood estimation based on an iterative Fisher scoring optimization (SAS statistical software, PROC LOGISTIC procedure). ${ }^{3}$ Part of the weight estimates for the link function are given in Table 1.

Contrast comparisons between a and $\mathbf{b}$ levels versus the other alignment conditions were all highly significant (two-tailed, $p<.0001$ ), and all pairwise comparisons within the other alignment conditions had high $p$ values. The estimates associated with $\mathbf{a}$ and $\mathbf{b}$ alignments were not significantly different in their absolute values. Disregarding the smaller interaction effects, one could state that local alignment enhanced the odds by a factor of almost three in the direction of the patch alignment orientation. Estimates for a second-order polynomial parameterization of the b-aligned condition separately, without any other effects, yielded estimates corresponding to the model $\ln [p(b) / p(a)]=1.0541-12.1539(|b| /|a|-1)+$ $5.5181(|b| /|a|-1)^{2}$. Calculation of an equilibrium point, 


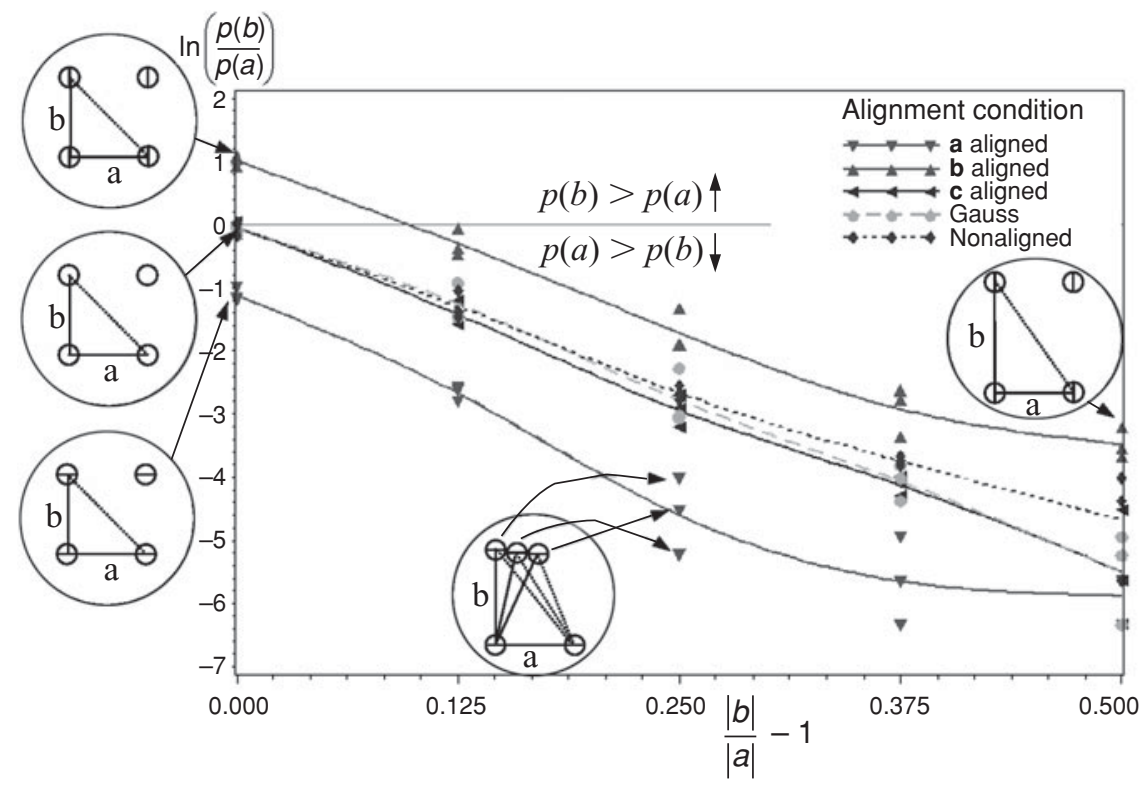

Figure 10. Plot of the log-odds of $b$ to a responses as a function of $|b| /|a|-1$ for the different alignment conditions. Schematic views of stimuli are connected with their corresponding data points; the line segments in the smallest circles indicate the local orientation of Gabor patches. Note that data points above 0 represent conditions in which more $b$ responses were given than a responses. Per level of $|b| /|a|-1$, there are three data points per alignment condition, because of the different lattice structures involved. The splines run through the average over the three lattice types within each combination of the other manipulations and connect averages across the distance ratios per level of the alignment manipulation.

where the attraction forces of alignment and proximity ratio are equally strong, is possible by solving the quadratic equation given above for $p(a)=p(b)$, or $\ln [p(b) /$ $p(a)]=0$. Applying this principle to the estimates for b-aligned Gabor patches, the interpolated solution is $|b| /|a|=1.09$. Some of the parameter estimates varied considerably over subjects; see Table 2 for an overview. The individual psychometric functions can be inspected in Figure 12. When the individual data sets for the naive subjects were included, the parameter estimates spanned a range of 0.6214 to $1.4891,-6.7635$ to -16.8092 , and -19.2559 to 14.5451 for the intercept, linear distance, and quadratic distance, respectively. The inset to Figure 12 provides a scatterplot of the intercept and the slope of quadratic approximation to the logit, with each plot symbol representing 1 subject. Our discussion of the issue of interindividual differences will be resumed later.

\section{Stepwise Logistic Modeling of Effects}

A further investigation of the role of the experimental manipulations and their interactions consisted of a stepwise forward-selection procedure. Alignment, distance, and lattice geometry, as well as their interactions, were assessed in terms of their advantage for logistic model fit and were added sequentially. Distance was treated as a class-level variable so as to keep the parametric shape of the proximity manipulation open. The significance of the score statistic related to each extension served as a criterion for priority of inclusion. The logistic regression parameters were reestimated after each iteration. This iterative procedure was ended when adding a term did not yield a significant (i.e., $p<.05$ ) increase of the score statistic.

The final model obtained with this procedure contained all main effects as well as their two-way interactions, except for the interaction between distance and alignment. The set of model variables as a whole was highly significant ( $p$ value well below .0001) in comparison with an intercept-only model (score $\chi^{2}=10,905$, likelihood ratio $\left.\chi^{2}(26)=10,842\right)$; in fact, the model already reached $p<.0001$ significance as soon as it included the distance ratio variable. The Wald $\chi^{2}$ values for the effects are informative as to their respective weights and importance in the final model fit (see Table 3). The interaction between lattice type and alignment, which was added as the last predictor to the model, had a $p$ value of about .022, which is only marginally significant. However, this interaction was quite consistent across observers, and it has an interesting interpretation (see below). There was a small but statistically significant role of lattice type in the results: Its main effect and significant interaction with distance, contributed by the responses from only 1 observer (Subject P.C.), were entirely due to the centered rectangular lattice geometries. This particular subject gave more b responses in these conditions for intermediate distance ratios in all alignment conditions.

The aforementioned variable selection procedure was also applied for each subject separately (i.e., for the 3 in- 

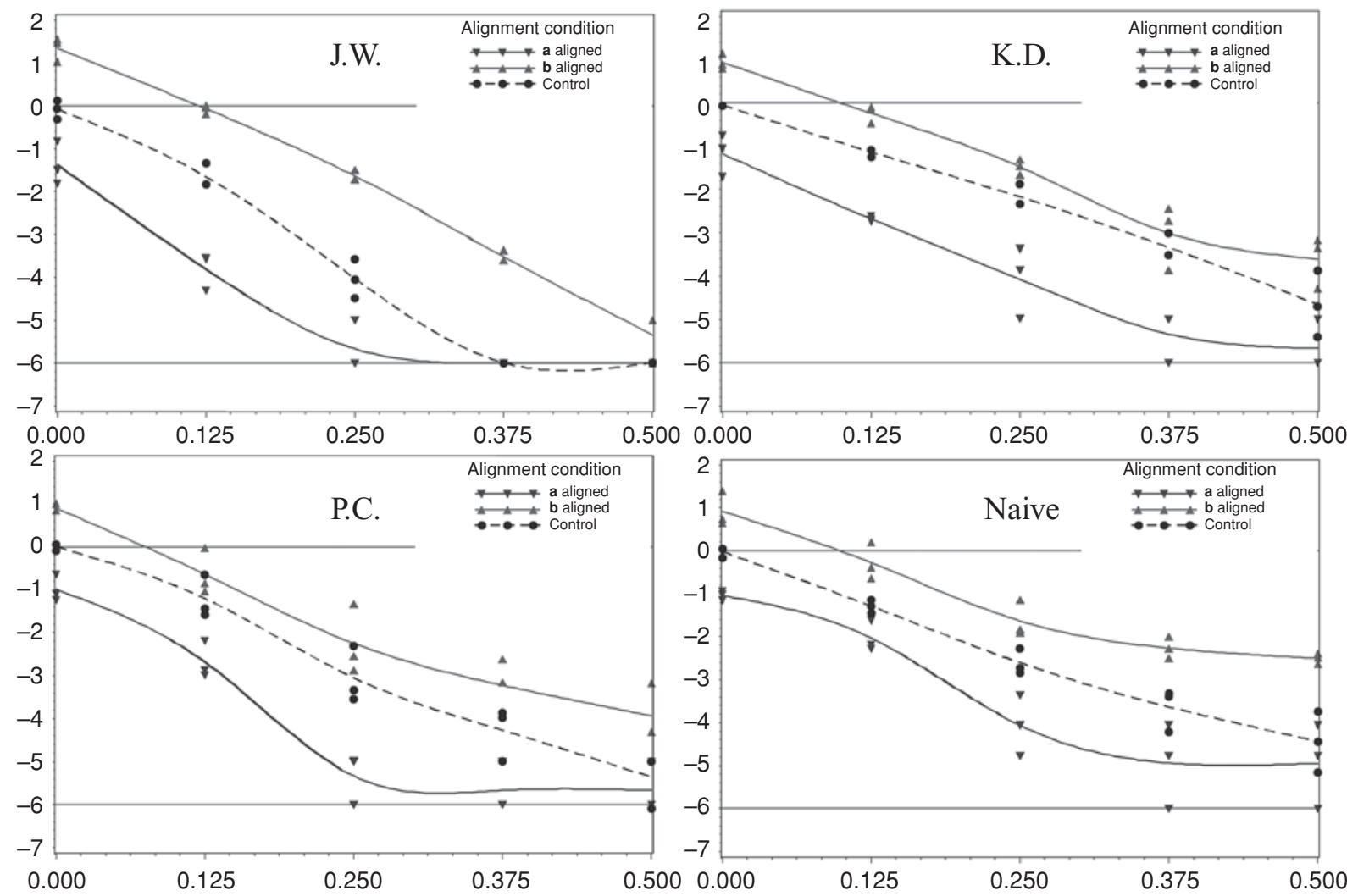

Figure 11. Plot of the log-odds of $b$ to a responses per subject. We grouped data over the control conditions for the sake of graph readability.

formed observers and the combined sessions of the naive subjects, or 4 "subjects" in total). The obtained models were not entirely consistent with respect to included interaction terms; however, these terms were always the last to join the model, because of their small effect size in comparison with the significant main effects. On the other hand, Wald $\chi^{2}$ statistics showed that both the effects of distance ratio and alignment were highly significant within each subject $(p<.0001)$. These effects were always the most powerful factors, with proximity always the strongest. This order of importance validates the robustness of the model obtained with the full data set as it applies to all subjects. Figure 13 depicts the evolution of the deviance $(-2 \times \log$-likelihood) when adding factors to the model. Although the differences in slopes indicate that the weights for the different model terms vary substantially across subjects, the relative rank order of the effects remained constant. One interaction, alignment $\times$ lattice type, appeared in the final forward-selection model for all subjects except P.C., although with small effect size. Within the different lattice types, comparatively more b responses were given for rectangular lattices that were aligned along the a vector, and fewer for rectangular lattices aligned along $\mathbf{b}$. This represents a relative grouping advantage for orientations orthogonal to the direction of alignment.

In sum, the most conspicuous results discussed here are the large effect sizes of both distance ratio and alignment

Table 1

Parameter Estimates for Main Effects in the Quadratic Proximity Model

\begin{tabular}{lrcr}
\hline \multicolumn{1}{c}{ Model Term for Parameter } & Estimate & Standard Error & Wald $\chi^{2}(1)$ \\
\hline Alignment: a aligned & -1.1000 & 0.0581 & $358.6586^{* * *}$ \\
Alignment: b aligned & 1.0541 & 0.0551 & $366.5008^{* * *}$ \\
Alignment: c aligned & -0.0066 & 0.0602 & $0.0119^{\text {n.s. }}$ \\
Alignment: Randomly aligned & -0.0139 & 0.0522 & $0.0711^{\text {n.s. }}$ \\
$|b| /|a|-1$ & -10.3020 & 0.6414 & $257.9999^{* * *}$ \\
$(|b| /|a|-1)^{2}$ & -1.3837 & 2.1305 & $0.4218^{\text {n.s. }}$ \\
\hline
\end{tabular}

Note-The condition with Gauss blob lattices is used as the reference level within the alignment class-level variable. ${ }^{* * *} p<.0001$. n.s., not significant, $p>.05$. 
Table 2

Quadratic Approximation of the Logit for the b-Aligned Gabor Lattices

\begin{tabular}{lcccc}
\hline \multicolumn{1}{c}{ Subject } & Intercept & $\begin{array}{c}\text { Linear } \\
\text { Estimate }\end{array}$ & $\begin{array}{c}\text { Quadratic } \\
\text { Estimate }\end{array}$ & $\begin{array}{c}\text { Equilibrium } \\
\text { Estimate }\end{array}$ \\
\hline J.W. & $1.3524^{* * *}$ & $-10.4868^{* * *}$ & $-6.2584^{\text {n.s. }}$ & 1.1203 \\
K.D. & $0.9559^{* * *}$ & $-10.1252^{* * *}$ & $1.4694^{\text {n.s. }}$ & 1.0957 \\
P.C. & $0.9254^{* * *}$ & $-14.0237^{* * *}$ & $8.8991^{*}$ & 1.0690 \\
Naive observers & $0.9398^{* * *}$ & $-12.2176^{* * *}$ & $10.3434^{* * *}$ & 1.0827 \\
\hline
\end{tabular}

Note-The intercept, linear, and quadratic estimates should not be compared with each other directly; their values weight different variable ranges. The last column shows the equilibrium point - that is, the value of $|b| /|a|$ for which there is a balance between proximity and alignment groupings, and hence where $p(a)=p(b)$ for the $\mathbf{b}$-aligned lattices. ${ }^{*} p<$ $.01 .{ }^{* * *} p<.0001$. n.s., not significant, $p>.05$.

and the consistency of their role as main effects across subjects, as shown by the data-driven variable selection procedure. The nonlinear nature of the influence of distance ratio on grouping of Gabor patches and the large interindividual differences in the weights of the various experimental manipulations on perceptual grouping were also obvious throughout the different analyses. The order of relative importance of the main effects was consistent across observers.

\section{DISCUSSION}

Irrespective of alignment condition, the log-odds of choosing the $\mathbf{b}$ alternative are a linear function of interelement distance over a large range of the distance manipulation. This finding is in line with results from previous research (Kubovy et al., 1998), and thus supports the validity of the pure distance law (Kubovy \& Wagemans, 1995) for Gabor lattices. In two respects, however, the data of the present study deviate from the pure distance law. First, for larger values, the relation between distance and perceptual grouping levels off for most Gabor lattice conditions, whereas local element alignment remains equally significant for all aligned lattice conditions. Second, the exploratory analysis revealed a weak, although fairly consistent, interaction between lattice geometry and alignment. The relatively large numbers of $\mathbf{b}$ responses for a-aligned rectangular lattices and of a responses for b-aligned rectangular lattices supports the existence of facilitation along a grouping axis orthogonal to the local element orientation. This result parallels those with sideby-side Gabor patches in the snake detection paradigm, as obtained by Field et al. (1993) and others (e.g., Bex et al., 2001), and is reminiscent of the contrast enhancement for orthogonal configurations reported by Polat and Sagi (1993).

For 1 subject (P.C.), the final forward-selection model had a slightly different composition than it did for the other subjects, in that it contained an effect of lattice type and an alignment $\times$ lattice type interaction. This is the result of a higher number of $\mathbf{b}$ responses for centered rectangular geometries with intermediate-distance ratios, where the a-grouping alternative normally dominates. Of all ge- ometries, centered rectangular lattices have the highest grouping entropy (Kubovy \& Wagemans, 1995). A possible explanation of the deviant pattern of results is more randomness in the data due to either a high threshold for use of the blank-response alternative or a large influence of prior orientation biases in conditions of high perceptual uncertainty for centered rectangular lattices.

The results obtained with the Gabor lattice paradigm allow for a precise evaluation of the relative importance of the factors involved in grouping, and they corroborate the conclusions from previous experiments on contour integration. At the same time, perceptual organization in Gabor lattices also sheds new light on theories of contour integration that have traditionally been developed to model contrast enhancement data or snake detection performance. The lattice method generates data that exhibit a lawful relationship between stimulus characteristics and a multistable, phenomenally coherent organization. This finding leads to a number of questions about how global organization relates to existing models. First, it is not obvious whether a one-layered network with integration mechanisms based solely on association field-type connectivity (Pettet et al., 1998; Yen \& Finkel, 1998) could explain why Gabor lattice organization is coherent and not partitioned - that is, why different dominant orientations do not occur in different parts of the lattice. This coherence feature of global organization requires integration of information spanning more than the classically assumed extent of an association field. Second, the popular explanation that relates local contrast enhancement to contour saliency (see, e.g., Usher et al., 1999) is not applicable to grouping in lattices. In the case of lattices, the spatial relations between elements are by definition invariant throughout the stimulus. Therefore, contrast enhancement or similar mechanisms would apply to the lattice as a whole and would not generate pop-out of one specific orientation. This does not mean that contrast modulation has no role to play in grouping phenomena. The situation with near-threshold contrast images or stimuli with a low signal-to-noise ratio might be different from perceptual organization in a suprathreshold regime. Although the primary task of the visual system in the former case is to identify whether or not any contour candidate is present, in the latter situation this system must decide between several competitive interpretations. In other words, the mechanisms and substrates for grouping might be subdivided in two distinct but interoperating systems: one

Table 3

Evaluation of Model Terms

\begin{tabular}{lcc}
\hline \multicolumn{1}{c}{ Effect } & $d f$ & Wald $\chi^{2}$ \\
\hline Distance & 4 & $5,542.4323^{* * *}$ \\
Alignment & 4 & $1,603.8621^{* * *}$ \\
Distance $\times$ lattice type & 8 & $31.5689^{* *}$ \\
Lattice type & 2 & $13.7658^{*}$ \\
Alignment $\times$ lattice type & 8 & $17.9322^{\dagger}$ \\
\hline${ }^{*} p<.01 .{ }^{* *} p<.001 .{ }^{* * *} p<.0001 . \quad{ }^{\dagger} p=.022$.
\end{tabular}




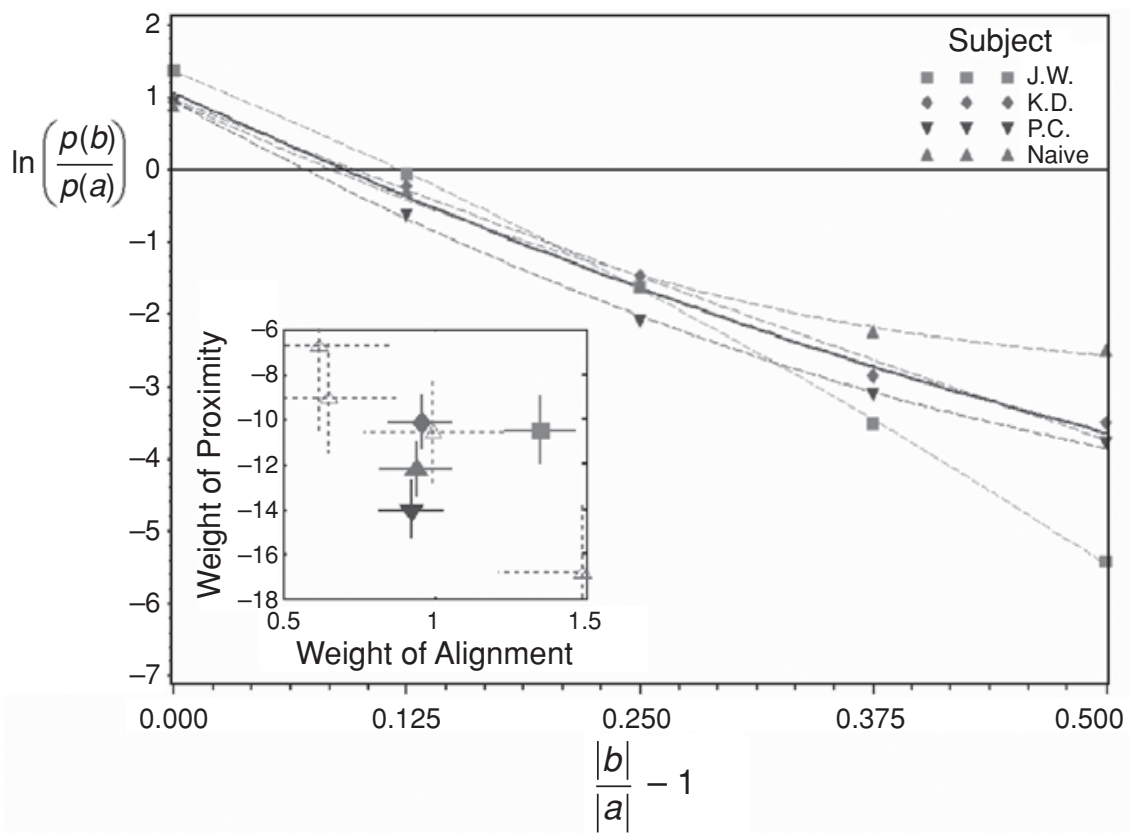

Figure 12. Plot of the log-odds of $b$ to a responses for the $b$-aligned condition, data aggregated over lattice type. The different dashed lines show the quadratic logistic model for the different subjects (see legend), and the solid line is the fit for the data merged across subjects. The inset scatterplot shows the weight of alignment (the intercept in the quadratic model, horizontal axis) and the corresponding linear weight for proximity (vertical axis), as reported in Table 2, per subject. The estimation derived from data merged over the naive subjects is shown as a filled upward-pointing triangle in the inset, and each of the individual naive subjects is represented as an unfilled triangle. Horizontal and vertical lines correspond to one standard error of estimate below and above the weight estimates.

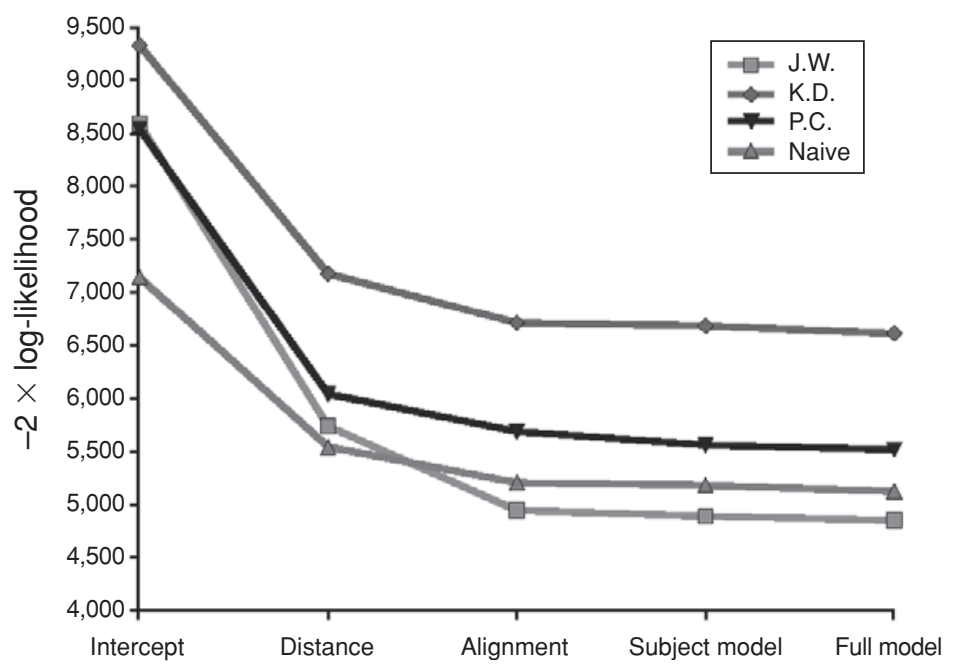

Figure 13. Interblock differences between effect deviance gains. Deviance ( $-2 \times \log$-likelihood) is a statistic measuring badness of fit; adding more model terms increases goodness of fit, and thus decreases the deviance. The downward slope can be considered to be the weight of the added term in explaining remaining variability in the log-odds of $b$ versus a responses. From left to right on the abscissa of this graph, distance ratio, alignment, further terms from the subject-specific forward-selection procedure ("Subject model"), and all other terms ("Full model") are added. The resulting deviance is plotted as a function of these model expansions. The deviance decrease obtained by adding the first term and, particularly, the second term varies across subjects. 
that identifies the presence or absence of a contour in a given location and orientation, and another that selects which contour elements are part of the same contour, in effect disambiguating a multistable organization into one solution. These systems seem to correspond largely to the frameworks of prespecified and dynamic grouping, respectively (see Watt \& Phillips, 2000). Neural models that concentrate on one of these aspects of grouping are bound to have different features or to exhibit different behaviors. Models that start by using correlated firing of neural units as a hypothesis for the emergence of dynamic binding (Choe \& Miikkulainen, 2004; Li, 1998; Yen \& Finkel, 1998) might have more potential as an explanation of transient global grouping (but note the critical evaluation by Shadlen \& Movshon, 1999, and the recent data on curve tracing by Roelfsema, Lamme, \& Spekreijse, 2004). In their specifications of neural architecture, the authors of these models often rely on the now well-established finding that long-range connections between pyramidal neurons in area V1 tend to connect groups of cells with a similar orientation tuning (Gilbert, 1992; Gilbert et al., 1996; Stettler et al., 2002). In the present study, all Gabor patches had the same orientation. Therefore, the stronger grouping along the vector with the same orientation as the local alignment necessitates the additional constraint that connections are more heavily weighted in orientation-selective cells that lie along the same global axis. Bosking et al. (1997; see also Chisum et al., 2003) have found a similar architecture of horizontal connections in tree shrew striate cortex, but it is currently unclear whether such an architecture is available in the striate cortex of other mammals.

The relative weights of feedback versus local interactions in the phenomena discussed here are still an important unresolved issue. Exactly the modulatory input from regions with larger receptive fields in higher layers might explain the spatial coherence in lattice stimuli. The finding that some subjects show an increased grouping tendency for parallel Gabor elements points to the need for research into the functional significance of psychophysically and neurophysiologically established facilitation along parallel contour elements that are not likely to belong to the same contour. The cases in which the logical solutions of contour integration and the observed phenomenal grouping diverge could potentially yield valuable information about the relative share of prespecified and dynamic aspects of grouping in visual experience.

In summary, we believe that the use of Gabor lattices in rigorous psychophysical experiments and analysis yields important prospects for the quantification of several grouping factors and their interactions and for further evaluation of current models of spatial integration. We propose the lattice paradigm as a complement to existing procedures, such as snake detection experiments, in order to establish different approaches to the problem of how to combine findings from psychophysics and the neurosciences into a unified account of perceptual grouping.

\section{REFERENCES}

BeAudot, W. H. A. (2002). Role of onset asynchrony in contour integration. Vision Research, 42, 1-9.

BeAudot, W. H. A., \& Mullen, K. T. (2003). How long range is contour integration in human color vision? Visual Neuroscience, 20, 51-64.

Beck, J., Rosenfeld, A., \& Ivry, R. (1989). Line segregation. Spatial Vision, 4, 75-101.

Ben-Av, M. B., \& SAgi, D. (1995). Perceptual grouping by similarity and proximity: Experimental results can be predicted by intensity autocorrelations. Vision Research, 35, 853-866.

Bex, P. J., Simmers, A. J., \& DAKIN, S. C. (2001). Snakes and ladders: The role of temporal modulation in visual contour integration. Vision Research, 41, 3775-3782.

Bosking, W., Zhang, Y., Schofield, B., \& FitzPatrick, D. (1997). Orientation selectivity and the arrangement of horizontal connections in tree shrew striate cortex. Journal of Neuroscience, 17, 2112-2127.

Bravais, M. A. (1866). Études cristallographiques [Crystallographic studies]. Paris: Gauthier-Villars. Electronic edition (1995) retrieved March 1, 2005, from http://gallica.bnf.fr/document?O=N096124.

Chisum, H. J., Mooser, F., \& FitzPatrick, D. (2003). Emergent properties of layer $2 / 3$ neurons reflect the collinear arrangement of horizontal connections in tree shrew visual cortex. Journal of Neuroscience, 23, 2947-2960.

Choe, Y., \& Mitikulainen, R. (2004). Contour integration and segmentation with self-organized lateral connections. Biological Cybernetics, 90, 75-88.

DAKIN, S. C., \& Hess, R. F. (1998). Spatial-frequency tuning of visual contour integration. Journal of the Optical Society of America, 15, 1486-1499.

Daugman, J. G. (1985). Entropy reduction and decorrelation in visual coding by oriented neural receptive fields. IEEE Transactions on Biomedical Engineering, 36, 107-114.

DRESP, B. (1999). Dynamic characteristics of spatial mechanisms coding contour structures. Spatial Vision, 12, 129-142.

Elder, J. H., \& GoldberG, R. M. (2002). Ecological statistics of Gestalt laws for the perceptual organization of contours. Journal of $\mathrm{Vi}$ sion, 2, 324-353.

Field, D. J., HaYes, A., \& Hess, R. F. (1993). Contour integration by the human visual system: Evidence for a local association field. Vision Research, 33, 173-193.

Field, D. J., Hayes, A., \& Hess, R. F. (2000). The roles of polarity and symmetry in the perceptual grouping of contour fragments. Spatial Vision, 13, 51-66.

FitzPATRICK, D. (2000). Seeing beyond the receptive field in primary visual cortex. Current Opinion in Neurobiology, 10, 438-443.

GILBERT, C. D. (1992). Horizontal integration and cortical dynamics. Neuron, 9, 1-13.

Gilbert, C. D., Das, A., Ito, M., Kapadia, M., \& Westheimer, G. (1996). Spatial integration and cortical dynamics. Proceedings of the National Academy of Sciences, 93, 615-622.

Hess, R. F., Beaudot, W. H. A., \& Mullen, K. T. (2001). Dynamics of contour integration. Vision Research, 41, 1023-1037.

Hess, R. F., \& DAKIN, S. C. (1999). Contour integration in the peripheral field. Vision Research, 39, 947-959.

Hess, R. F., Dakin, S. C., \& Field, D. J. (1998). The role of "contrast enhancement" in the detection and appearance of visual contours. Vision Research, 38, 783-787.

HEss, R. [F.], \& FIELD, D. (1999). Integration of contours: New insights. Trends in Cognitive Sciences, 3, 480-486.

Hess, R. F., HaYes, A., \& Field, D. J. (2003). Contour integration and cortical processing. Journal of Physiology, 97, 105-119.

Kapadia, M. K., Ito, M., Gilbert, C. D., \& Westheimer, G. (1995). Improvement in visual sensitivity by changes in local context: Parallel studies in human observers and in V1 of alert monkeys. Neuron, 15, 843-856.

Kapadia, M. K., Westheimer, G., \& Gilbert, C. D. (2000). Spatial distribution of contextual interactions in primary visual cortex and in visual perception. Journal of Neurophysiology, 84, 2048-2062. 
Kovacs, I., \& Julesz, B. (1993). A closed curve is much more than an incomplete one: Effect of closure in figure-ground segmentation. Proceedings of the National Academy of Sciences, 90, 7495-7497.

Kubovy, M. (1994). The perceptual organization of dot lattices. Psychonomic Bulletin \& Review, 1, 182-190.

Kubovy, M., Holcombe, A. O., \& Wagemans, J. (1998). On the lawfulness of grouping by proximity. Cognitive Psychology, 35, 71-98.

Kubovy, M., \& Wagemans, J. (1995). Grouping by proximity and multistability in dot lattices: A quantitative Gestalt theory. Psychological Science, 6, 225-234.

LI, Z. (1998). A neural model of contour integration in the primary visual cortex. Neural Computation, 10, 903-940.

LUCE, R. D. (1959). Individual choice behavior: A theoretical analysis. New York: Wiley.

Marcelja, S. (1980). Mathematical description of the responses of simple cortical cells. Journal of the Optical Society of America, 70, 1297-1300.

McIlhagGa, W. H., \& Mullen, K. T. (1996). Contour integration with colour and luminance contrast. Vision Research, 36, 1265-1279.

Meese, T. S., Hess, R. F., \& Williams, C. B. (2001). Spatial coherence does not affect contrast discrimination for multiple Gabor stimuli. Perception, 30, 1411-1422.

Nugent, A. K., Keswani, R. N., Woods, R. L., \& Peli, E. (2003). Contour integration in peripheral vision reduces gradually with eccentricity. Vision Research, 43, 2427-2437.

Pettet, M. W., McKee, S. P., \& Grzywacz, N. M. (1998). Constraints on long range interactions mediating contour detection. Vision Research, 38, 865-879.

Polat, U. (1999). Functional architecture of long-range perceptual interactions. Spatial Vision, 12, 143-162.

Polat, U., Mizobe, K., Pettet, M. W., Kasamatsu, T., \& Norcia, A. M. (1998). Collinear stimuli regulate visual responses depending on cell's contrast threshold. Nature, 391, 580-584.

Polat, U., \& Norcia, A. M. (1996). Neurophysiological evidence for contrast dependent long-range facilitation and suppression in the human visual cortex. Vision Research, 36, 2099-2109.

Polat, U., \& SAGI, D. (1993). Lateral interactions between spatial channels: Suppression and facilitation revealed by lateral masking experiments. Vision Research, 33, 993-999.

Polat, U., \& Sagi, D. (1994). Spatial interactions in human vision: From near to far via experience-dependent cascades of connections. Proceedings of the National Academy of Sciences, 91, 1206-1209.

Quinlan, P. T., \& Wilton, R. N. (1998). Grouping by proximity or similarity? Competition between the Gestalt principles in vision. Perception, 27, 417-430.

Roelfsema, P. R., Lamme, V. A. F., \& Spekreisse, H. (1998). Objectbased attention in the primary visual cortex of the macaque monkey. Nature, 395, 376-381.

Roelfsema, P. R., Lamme, V. A. F., \& Spekreijse, H. (2004). Synchrony and covariation of firing rates in the primary visual cortex during contour grouping. Nature Neuroscience, 7, 982-991.

Schumann, F. (1900). Beiträge zur Analyse der Gesichtswahrnehmungen: 1. Einige Beobachtungen über die Zusammenfassung von Gesichtseindrücken zu Einheiten [Contributions to the analysis of visual perceptions: 1 . Some observations on the grouping of visual impressions into units]. Zeitschrift für Psychologie \& Physiologie der Sinnesorgane, 23, 1-32.

Shadlen, M. N., \& Movshon, J. A. (1999). Synchrony unbound: A critical evaluation of the temporal binding hypothesis. Neuron, 24, 67-77.

Sigman, M., Cecchi, G. A., Gilbert, C. D., \& Magnasco, M. O. (2001). On a common circle: Natural scenes and Gestalt rules. Proceedings of the National Academy of Sciences, 13, 1935-1940.
Smits, J. T. S., Vos, P. G., \& Van Oeffelen, M. P. (1985). The perception of a dotted line in noise: A model of good continuation and some experimental results. Spatial Vision, 1, 163-177.

Stettler, D. D., Das, A., Benett, J., \& Gilbert, C. D. (2002). Lateral connectivity and contextual interactions in macaque primary visual cortex. Neuron, 36, 739-750.

Tzevanov, T., \& Dresp, B. (2002). Short- and long-range effects in line contrast integration. Vision Research, 42, 2493-2498.

Usher, M., Bonneh, Y., SAGi, D., \& Herrmann, M. (1999). Mechanisms for spatial integration in visual detection: A model based on lateral interactions. Spatial Vision, 12, 187-209.

UtTal, W. R. (1975). An autocorrelation theory of form detection. Hillsdale, NJ: Erlbaum.

Wagemans, J., Claessens, P., Delbarge, K., Eycken, A., \& Kubovy, M. (1999, August). Perceptual organization of Gabor lattices: Relations between Gestalt principles of grouping-by-proximity and groupingby-similarity. Paper presented at the European Conference on Visual Perception (ECVP), Trieste, Italy.

Wagemans, J., Eycken, A., Claessens, P., \& Kubovy, M. (1999, May). Interactions between grouping principles in Gabor lattices: Proximity and orientation alignment. Poster session presented at the Annual Meeting of the Association for Research in Vision and Ophthalmology (ARVO), Fort Lauderdale, FL.

Watt, R. J., \& Phillips, W. A. (2000). The function of dynamic grouping in vision. Trends in Cognitive Sciences, 4, 447-454.

Wertheimer, M. (1955). Laws of organization in perceptual forms. In W. D. Ellis (Ed.), A source book of Gestalt psychology (pp. 71-88). London: Routledge \& Kegan Paul. (Original work published 1923)

Yen, S., \& FinKeL, L. H. (1998). Extraction of perceptually salient contours by striate cortical networks. Vision Research, 38, 719-741.

Zucker, S. W., Stevens, K. A., \& Sander, P. (1983). The relation between proximity and brightness similarity in dot patterns. Perception \& Psychophysics, 34, 513-522.

\section{NOTES}

1. Concerning this point, we note that in pilot experiments (Wagemans, Eycken, Claessens, \& Kubovy, 1999) no effect of dot polarity was found

2. We do not consider data from 1 naive observer who gave only responses corresponding to the local Gabor orientations or the "blank" option; it is very likely that he misunderstood the task instructions. For most within-subjects analyses, we aggregated the data across naive subjects to obtain statistical power comparable to that for the data sets of the informed subjects. This is justified because the results from the individual naive subjects were not structurally different from each other or from the data sets generated by the informed observers.

3 . We also applied an analysis based on an underlying extreme-value distribution with a linear equation of the independent variables (also known as a complementary log-log model) rather than a logistic distribution in which the log-odds follow a quadratic trend. In the latter scheme, one more parameter was fitted. The Schwarz criterion for goodness of fit, which corrects for number of parameters, had similar values for both approaches. We preferred the quadratic logistic model for its link with the pure distance model (Kubovy \& Wagemans, 1995) and the interpretability of the parameters.

(Manuscript received November 8, 2004; revision accepted for publication February 28, 2005.) 